\title{
Excluding a Bipartite Circle Graph from Line Graphs
}

Sang-il Oum*

DEPARTMENT OF MATHEMATICAL SCIENCES

KAIST, DAEJEON 305-701

REPUBLIC OF KOREA

E-mail: sangil@kaist.edu

Received July 5, 2006; Revised July 2, 2008

Published online 27 August 2008 in Wiley InterScience(www.interscience.wiley.com).

DOI 10.1002/jgt.20353

\begin{abstract}
We prove that, for a fixed bipartite circle graph $H$, all line graphs with sufficiently large rank-width (or clique-width) must have a pivotminor isomorphic to $H$. To prove this, we introduce graphic delta-matroids. Graphic delta-matroids are minors of delta-matroids of line graphs and they generalize graphic and cographic matroids. @ 2008 Wiley Periodicals, Inc. J Graph Theory 60: 183-203, 2009
\end{abstract}

Keywords: rank-width; circle graph; line graph; pivot-minor; graphic delta-matroid; tree-width

\section{INTRODUCTION}

Robertson and Seymour [18] proved that every graph of sufficiently large treewidth must contain a minor isomorphic to a fixed planar graph. Their theorem was generalized to a theorem on representable matroids by Geelen et al. [8], stating that every matroid representable over a fixed finite field of sufficiently large

Contract grant sponsor: NSF; Contract grant number: 0354742; Contract grant sponsor: SRC Program of Korea Science and Engineering Foundation (KOSEF); Contract grant number: R11-2007-035-01002-0.

*The research was partially done while the author was at Georgia Institute of Technology.

Journal of Graph Theory

(c) 2008 Wiley Periodicals, Inc. 

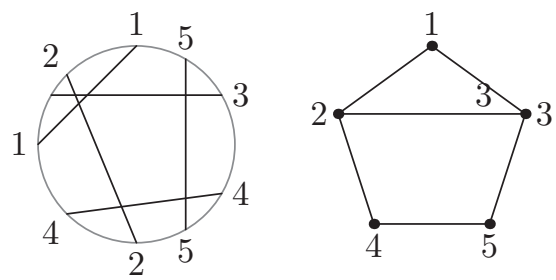

FIGURE 1. Chord diagrams and circle graphs.

branch-width must contain a minor isomorphic to a fixed planar matroid. (A planar matroid is the cycle matroid of a planar graph.)

We propose the following conjecture, which is another generalization of Robertson and Seymour's grid theorem. Rank-width is a graph width parameter, like tree-width, introduced by Oum and Seymour [16] to investigate clique-width [4]. Pivot-minors of a graph $G$ are graphs obtained from $G$ by repeatedly applying certain operations, like minors [15]. We defer definitions of rank-width and pivotminor to Section 2. A circle graph is the intersection graph of chords in a circle, see Figure 1. Lemma 8.2 states a result of de Fraysseix [5] that relates bipartite circle graphs to planar graphs.

Conjecture 1.1. Let H be a bipartite circle graph. Every graph $G$ with sufficiently large rank-width must have a pivot-minor isomorphic to $H$.

Surprisingly this conjecture would, if true, imply Robertson and Seymour's grid theorem as well as its matroidal version for binary matroids of Geelen et al. This conjecture is still open. Using the relation between bipartite graphs and binary matroids, Oum [15] showed that when $G$ is bipartite, the conjecture is implied by the grid theorem for binary matroids of Geelen et al. We will also discuss in Section 9 that, when $G$ is a circle graph, the conjecture is implied by a theorem in the $\mathrm{PhD}$ thesis of Johnson [11] on 4-regular Eulerian digraphs.

Our main theorem is that the conjecture is true when $G$ is a line graph. It is known that the rank-width of a family of line graphs is bounded if and only if the tree-width of their primal graphs is bounded, see, for instance [9]. So ultimately we apply Robertson and Seymour's grid theorem on the primal graphs. But the difficulty arises because a pivot-minor of a line graph may not be a line graph at all. Compare this to the fact that both bipartite graphs and circle graphs are closed under taking pivot-minors.

To overcome this, we define a graphic delta-matroid. Delta-matroids are generalizations of matroids introduced by Bouchet [1]. While matroids capture combinatorial properties on linear independence of column vectors of matrices, delta-matroids capture combinatorial properties on nonsingular principal submatrices of skew-symmetric or symmetric matrices. Delta-matroids that are representable by skew-symmetric matrices over $\mathrm{GF}(2)$ are called even binary deltamatroids. Since skew-symmetric matrices over GF(2) are equivalent to simple graphs, we observe that even binary delta-matroids naturally correspond to simple 
graphs, which we call fundamental graphs of delta-matroids. Graphic deltamatroids are defined in such a way that pivot-minors of line graphs are exactly fundamental graphs of graphic delta-matroids. So far this is a vague explanation and the details will be discussed later.

We define graphic delta-matroids from a graft, which is a pair $(G, T)$ of a graph $G$ and a subset $T$ of $V(G)$ (Section 4). We will show that graft minors naturally correspond to delta-matroid minors (Section 5) and if the branch-width of the graph in a graft is small, then the rank-width of a fundamental graph of the graphic delta-matroid given by the graft is small (Section 6). Then we use Robertson and Seymour's grid theorem on the graph in a graft and deduce our main theorem (Sections 7 and 8).

\section{PRELIMINARIES}

In our article, graphs may have parallel edges or loops. A graph is simple if it has no loops and no parallel edges. All graphs in this article are finite.

For an $A \times B$ matrix $M=\left(a_{i j}\right)_{i \in A, j \in B}$ and subsets $X$ of $A$ and $Y$ of $B$, let $M[X, Y]$ be the $X \times Y$ submatrix of $M$, that is, $\left(a_{i j}\right)_{i \in X, j \in Y}$ and let $M[X]=M[X, X]$. The adjacency matrix $A(G)$ of a graph $G=(V, E)$ is a $V \times V$ 0-1 matrix $\left(a_{i j}\right)_{i, j \in V}$ over the binary field $\mathrm{GF}(2)$ such that $a_{i j}=1$ if $i j \in E$ and $a_{i j}=0$ otherwise.

Rank-width and branch-width. For a graph $G$ and a subset $F$ of $E(G)$, let $\beta_{G}(F)$ be the number of vertices incident to both $F$ and $E(G) \backslash F$. For a simple graph $G$ and a subset $X$ of $V(G)$, let $\rho_{G}(X)$ be $\operatorname{rank}(A(G)[X, V(G) \backslash X])$, called the cut-rank function. Note that when measuring the matrix rank, the matrix $A(G)[X, V(G) \backslash X]$ is considered over $\operatorname{GF}(2)$ because we define $A(G)$ as a matrix over $\mathrm{GF}(2)$. Both $\beta_{G}$ and $\rho_{G}$ are symmetric and submodular; in other words,

$$
\begin{aligned}
\beta_{G}(F) & =\beta_{G}(E(G) \backslash F), & & \text { for all } F \subseteq E(G), \\
\beta_{G}\left(F_{1}\right)+\beta_{G}\left(F_{2}\right) & \geq \beta_{G}\left(F_{1} \cap F_{2}\right)+\beta_{G}\left(F_{1} \cup F_{2}\right), & & \text { for all } F_{1}, F_{2} \subseteq E(G), \\
\rho_{G}(X) & =\rho_{G}(V(G) \backslash X), & & \text { for all } X \subseteq V(G), \\
\rho_{G}\left(X_{1}\right)+\rho_{G}\left(X_{2}\right) & \geq \rho_{G}\left(X_{1} \cap X_{2}\right)+\rho_{G}\left(X_{1} \cup X_{2}\right) . & & \text { for all } X_{1}, X_{2} \subseteq V(G) \text { (see [16]). }
\end{aligned}
$$

Let $\mathbb{Z}$ be the set of integers. For a symmetric submodular function $f: 2^{V} \rightarrow \mathbb{Z}$ on a finite set $V$, the branch-width is defined as follows. A tree is subcubic if all vertices have degree 1 or 3. A branch-decomposition of the symmetric submodular function $f$ is a pair $(T, \mu)$ consisting of a subcubic tree $T$ and a bijective function $\mu: V \rightarrow\{t: t$ is a leaf of $T\}$. (If $|V| \leq 1$ then $f$ admits no branch-decomposition.) For an edge $e$ of $T$, the connected components of $T \backslash e$ induce a partition $(X, Y)$ of the set of leaves of $T$. The width of an edge $e$ of a branch-decomposition $(T, \mu)$ is $f\left(\mu^{-1}(X)\right)$. The width of $(T, \mu)$ is the maximum width of all edges of $T$. The branch-width of $f$ is the minimum of the width of all branch-decompositions of $f$. (If $|V| \leq 1$, we define the branch-width of $f$ to be $f(\emptyset)$.) 
Branch-width of graphs was defined by Robertson and Seymour [18]. The branch-width and a branch-decomposition of a graph $G$ are defined to be the branchwidth and a branch-decomposition, respectively, of $\beta_{G}$. Similarly, when $G$ is simple, Oum and Seymour [16] defined the rank-width and a rank-decomposition of $G$ to be the branch-width and a branch-decomposition, respectively, of $\rho_{G}$. Rank-width is related to clique-width (introduced by Courcelle and Olariu [4]) in the following manner: rank-width is at most clique-width, and clique-width is at most $2^{k+1}-1$ in which $k$ is the rank-width [16].

Matroids. Let us review matroid theory. For general matroid theory, we refer to Oxley's book [17]. We call $\mathcal{M}=(E, \mathcal{I})$ a matroid if $E$ is a finite set and $\mathcal{I}$ is a collection of subsets of $E$ satisfying the following three axioms.

(i) $\emptyset \in \mathcal{I}$.

(ii) If $A \in \mathcal{I}$ and $B \subseteq A$, then $B \in \mathcal{I}$.

(iii) For every subset $Z$ of $E$, all maximal subsets of $Z$ in $\mathcal{I}$ have the same size $r(Z)$. We call $r(Z)$ the rank of $Z$.

A subset of $E$ contained in $\mathcal{I}$ is called independent in $\mathcal{M}$. We let $E(\mathcal{M})=E$. A base is a maximal independent set. A matroid $\mathcal{M}=(E, \mathcal{I})$ is binary if there exists a matrix $N$ over GF(2) whose columns are indexed by $E$ such that $\mathcal{I}$ is the collection of subsets of $E$ whose corresponding columns are linearly independent. The connectivity function $\lambda_{\mathcal{M}}$ of $\mathcal{M}$ is $\lambda_{\mathcal{M}}(X)=r(X)+r(E \backslash X)-r(E)+1$. The branch-width and a branch-decomposition of a matroid $\mathcal{M}$ are defined to be the branch-width and a branch-decomposition, respectively, of $\lambda_{\mathcal{M}}$.

For a graph $G$, the cycle matroid $\mathcal{M}(G)$ is the matroid on $E(G)$ where a set $F$ of edges is independent if and only if $F$ induces no cycles in $G$. The fundamental graph of a matroid $\mathcal{M}=(E, \mathcal{I})$ with respect to a base $B$ is the bipartite graph on the vertex set $E$ such that $a \in B, b \notin B$ are adjacent if and only if $(B \backslash\{a\}) \cup\{b\}$ is independent in $\mathcal{M}$. Oum [15] showed that the rank-width of a fundamental graph of a binary matroid $\mathcal{M}$ is exactly one less than the branch-width of $\mathcal{M}$.

Delta-matroids. A matrix $A$ is symmetric if $A^{t}=A$, and skew-symmetric if $A^{t}=-A$ and the diagonal entries are zero. Let $V$ be a finite set. For a $V \times V$ skew-symmetric or symmetric matrix $A$ over a field $F$, let $\mathcal{F}(A)=\{X \subseteq V$ : $A[X]$ is nonsingular $\}$. We assume that $A[\emptyset]$ is nonsingular. For two sets $A$ and $B$, let $A \Delta B=(A \backslash B) \cup(B \backslash A)$. Bouchet [2] showed that $\mathcal{F}=\mathcal{F}(A)$ satisfies the symmetric exchange axiom:

If $X, Y \in \mathcal{F}$ and $x \in X \Delta Y$, then there is $y \in X \Delta Y$ such that $X \Delta\{x, y\} \in \mathcal{F}$.

A delta-matroid is a pair $(V, \mathcal{F})$ consisting of a finite set $V$ and a nonempty collection $\mathcal{F}$ of subsets of $V$ satisfying (1). A subset of $E$ contained in $\mathcal{F}$ is called feasible. If $\mathcal{M}=(V, \mathcal{F})$ is a delta-matroid, then for a subset $X$ of $V$, we can obtain another 
delta-matroid $\mathcal{M} \Delta X=(V, \mathcal{F} \Delta X)$ where $\mathcal{F} \Delta X=\{F \Delta X: F \in \mathcal{F}\}$. This operation is called twisting. A delta-matroid $\mathcal{M}=(V, \mathcal{F})$ is representable over a field $F$ if there exist a skew-symmetric or symmetric matrix $A$ over $F$ and a set $X$ such that $\mathcal{F}=\mathcal{F}(A) \Delta X$. A delta-matroid is binary if it is representable over $\operatorname{GF}(2)$. A deltamatroid is even if $|X \Delta Y|$ is even for all feasible sets $X$ and $Y$. Consequently a delta-matroid $\mathcal{M}=(V, \mathcal{F})$ is an even binary delta-matroid if and only if there is a skew-symmetric matrix $A$ over $\mathrm{GF}(2)$ and a subset $X$ of $V$ such that $\mathcal{F}=\mathcal{F}(A) \Delta X$. A simple graph having $A$ as the adjacency matrix is called a fundamental graph of the even binary delta-matroid $\mathcal{M}$. It is easy to construct a fundamental graph of an even binary delta-matroid: pick a feasible set $X$ and construct a simple graph on $V$ such that two vertices $a, b \in V$ are adjacent if and only if $X \Delta\{a, b\}$ is feasible.

If there is a feasible subset of $V \backslash X$, then $\mathcal{M} \backslash X=(V \backslash X,\{F: F \in \mathcal{F}, F \cap$ $X=\emptyset\})$ is a delta-matroid. This operation is called deletion. A minor of a deltamatroid $\mathcal{M}$ is a delta-matroid obtained from $\mathcal{M}$ by repeatedly applying twisting and deletion.

Pivoting. For a symmetric or skew-symmetric matrix $A$, if $A$ is partitioned as

$$
A=\begin{gathered}
X \\
Y \\
Y
\end{gathered}\left(\begin{array}{ll}
\alpha & \beta \\
\gamma & \delta
\end{array}\right)
$$

and $A[X]=\alpha$ is nonsingular, then let

$$
A * X=\begin{gathered}
X \\
Y
\end{gathered}\left(\begin{array}{cc}
X & Y \\
\alpha^{-1} & \alpha^{-1} \beta \\
-\gamma \alpha^{-1} & \delta-\gamma \alpha^{-1} \beta
\end{array}\right)
$$

This operation is called pivoting. Tucker [20] showed that, when $A[X]$ is nonsingular, $A * X[Y]$ is nonsingular if and only if $A[X \Delta Y]$ is nonsingular. Consequently if $\mathcal{M}=(V, \mathcal{F}(A))$ is a delta-matroid and $X$ is a feasible set of $\mathcal{M}$, then $\mathcal{M} \Delta X=(V, \mathcal{F}(A * X))$.

This implies that all fundamental graphs of an even binary delta-matroid are obtainable from one fundamental graph by pivoting (in the adjacency matrix). In addition, all fundamental graphs of an even binary delta-matroid are generated by repeatedly pivoting pairs of adjacent vertices, because every $k \times k$ nonsingular skew-symmetric matrix has a $2 \times 2$ nonsingular principal submatrix if $k \geq 2$. So when considering fundamental graphs of even binary delta-matroids, it is enough to consider the pivoting operations on a pair of vertices. Let $G \wedge u v$ denote the simple graph obtained from a simple graph $G$ by pivoting a pair of adjacent vertices 

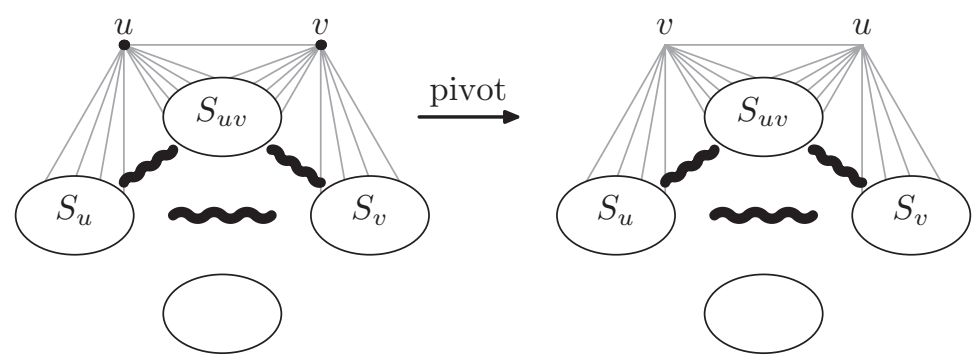

Figure 2. Pivoting (edges between three sets are 'toggled' and $u$, vare switched).

$u, v$ of $G$ in the adjacency matrix. This operation is also called pivoting an edge of a simple graph.

We can describe $G \wedge u v$ as follows. Let $G=(V, E)$ be a simple graph. For disjoint subsets $S, T$ of $V$, let $[S, T]=\{s t: s \in S, t \in T\}$. Let $S_{u}$ be the set of vertices (other than $u, v$ ) that are adjacent to $u$ but nonadjacent to $v$. Let $S_{v}$ be the set of vertices (other than $u, v$ ) that are adjacent to $v$ but nonadjacent to $u$. Let $S_{u v}$ be the set of common neighbors of $u$ and $v$. Let $G^{\prime}=\left(V, E \Delta\left[S_{u}, S_{v}\right] \Delta\left[S_{u}, S_{u v}\right] \Delta\left[S_{v}, S_{u v}\right]\right)$. Then $G \wedge u v$ is the graph obtained from $G^{\prime}$ by swapping the labels of $u$ and $v$. See Figure 2.

Minors of delta-matroids and pivot-minors of graphs. A pivot-minor of a simple graph $G$ is a graph obtained from $G$ by repeatedly applying the operations of pivoting edges and deleting vertices. Pivoting preserves the rank-width [15] and therefore the rank-width of a pivot-minor of a simple graph $G$ is at most the rankwidth of $G$. Note that pivot-minors of bipartite graphs are bipartite and pivot-minors of circle graphs are circle graphs, see Bouchet [3]. (Pivot-minors were called $p$ reductions by Bouchet [3].)

We will now explain why pivot-minors of fundamental graphs of an even binary delta-matroid are fundamental graphs of minors of the delta-matroid. Suppose that $\mathcal{M}$ is an even binary delta-matroid and $\mathcal{M}^{\prime}=\mathcal{M} \Delta X \backslash Y$ is a minor of $\mathcal{M}$. We may assume that $\mathcal{M}=(V, \mathcal{F}(A))$ for a skew-symmetric matrix $A$ over $\mathrm{GF}(2)$, which is the adjacency matrix of a fundamental graph $G$. If $Y=\emptyset$, then $\mathcal{M}^{\prime}$ is an even binary delta-matroid and its fundamental graph can be obtained from a fundamental graph of $\mathcal{M}$ by pivoting as discussed above. If $Y$ is nonempty, we may assume that $|Y|=1$ by the induction argument. Let $Y=\{v\}$. If $v \notin X$, then $\mathcal{M}^{\prime}=(V \backslash\{v\}, \mathcal{F}(A[V \backslash$ $\{v\}]) \Delta X)$ and therefore $G \backslash v$ is a fundamental graph of $\mathcal{M}^{\prime}$. If $v \in X$, then there is a feasible set $Z$ in $\mathcal{M}$ containing $v$, otherwise $\mathcal{M} \Delta X \backslash Y$ is not defined. We can pick $w \in Z$ so that $w \neq v$ and $\{v, w\}$ is feasible. Then $\mathcal{M}^{\prime}=(\mathcal{M} \Delta\{v, w\} \backslash$ $\{v\}) \Delta(X \Delta\{v, w\})$ and $G \wedge v w \backslash v$ is a fundamental graph of $\mathcal{M}^{\prime}$. We conclude that pivot-minors of simple graphs exactly correspond to minors of even binary deltamatroids. This also implies that minors of even binary delta-matroids are even binary. In fact, minors of representable or binary delta-matroids are representable or binary, respectively, as was shown by Bouchet [2]. 


\section{ADJACENCY MATRICES OF LINE GRAPHS}

The line graph $L(G)$ of a graph $G$ is the simple graph whose vertices are edges of $G$ such that two distinct edges $e, f$ of $G$ are adjacent in the line graph if and only if $e$ and $f$ share at least one end in G. Kishi and Uetake [13] proved the following theorem, which later appeared in the articles by Doob [7] and Deo et al. [6].

Theorem 3.1 (Kishi and Uetake [13]). The adjacency matrix of the line graph of a simple graph $G$ is nonsingular if and only if every component of $G$ is a tree with an odd number of vertices.

In order to extend Theorem 3.1 to non-simple graphs, we define the binary line graph $B L(G)$ of a graph $G$ to be the simple graph on $E(G)$ such that two distinct edges $e, f$ of $G$ are adjacent in $B L(G)$ if and only if both $e$ and $f$ are non-loops and $e$ and $f$ share exactly one end. We note that if $G$ is simple, then $L(G)=B L(G)$.

Proposition 3.2. The adjacency matrix of the binary line graph of a graph $G$ is nonsingular if and only if every component of $G$ is a tree with an odd number of vertices.

Proof. If every component of $G$ is a tree with an odd number of vertices, then $G$ is simple. Conversely, if $A(B L(G))$ is nonsingular, then $G$ is simple, because otherwise $A(B L(G))$ has a row with zeros or two identical rows. Now this proposition follows from Theorem 3.1.

\section{GRAPHIC DELTA-MATROIDS FROM GRAFTS}

Let $G=(V, E)$ be a graph and let $T$ be a set of vertices of $G$. Recall that graphs may have loops or parallel edges. A set $X$ spans a graph $G$ if $V(G)=X$. A subgraph $H$ of $G$ is called a $T$-spanning subgraph of $G$ if $V(G)=V(H)$ and for each component $C$ of $H$, either

(i) $|V(C) \cap T|$ is odd, or

(ii) $V(C)$ spans a component without a vertex in $T$, of $G$.

A graft is a pair $(G, T)$ consisting of a graph $G$ and a set $T$ of vertices of $G$. A set $F$ of edges of $G$ is feasible in $(G, T)$ if it is the edge-set of a $T$-spanning forest of $G$. Let $\mathcal{G}(G, T)=(E(G), \mathcal{F})$ such that $\mathcal{F}$ is the set of all feasible sets of $(G, T)$. A delta-matroid $\mathcal{M}$ is graphic if there exist a graft $(G, T)$ and a set $X$ of edges of $G$ such that $\mathcal{M}=\mathcal{G}(G, T) \Delta X$. We will first prove that $\mathcal{G}(G, T)$ is a delta-matroid.

Proposition 4.1. Let $(G, T)$ be a graft. Then $\mathcal{G}(G, T)$ is a delta-matroid.

Proof. Let $V=V(G)$. We may assume that $G$ is connected, because, if $X_{i}$ is an feasible set in the $i$ th component of $(G, T)$, then the disjoint union of $X_{i}$ 's is feasible in $(G, T)$. We may assume that $|T| \geq 1$, because otherwise adding one vertex into $T$ does not change $\mathcal{G}(G, T)$. It is easy to show the existence of a Journal of Graph Theory DOI 10.1002/jgt 
feasible set in $(G, T)$, by taking a spanning tree and removing an appropriate edge if necessary.

Let $X, Y$ be feasible sets in $(G, T)$ and let $e \in X \Delta Y$. We claim that there exists an edge $f$ of $G$ in $X \Delta Y$ such that $X \Delta\{e, f\}$ is feasible in $(G, T)$.

Let us first assume that $e \in X$. Let $C$ be the component of the subgraph $(V, X)$ containing $e$. Since $X$ is feasible, $|V(C) \cap T|$ is odd and therefore the subgraph ( $V, X \backslash\{e\})$ has a unique component $C_{1}$ such that $\left|V\left(C_{1}\right) \cap T\right|$ is even. If $Y$ has an edge $f$ joining a vertex of $C_{1}$ to a vertex not in $C_{1}$, then $X \Delta\{e, f\}$ is feasible because each component of $(V, X \backslash\{e\})$ except $C_{1}$ has an odd number of vertices in $T$. So we may assume that no edges in $Y$ join $C_{1}$ to outside. In particular this implies that $V\left(C_{1}\right) \cap T \neq \emptyset$. Then there is a list of components $D_{1}, \ldots, D_{m}$ of the subgraph $(V, Y)$ such that $V\left(D_{1}\right) \cup \cdots \cup V\left(D_{m}\right)=V\left(C_{1}\right)$. Each $D_{i}$ has an odd number of vertices in $T$ because $Y$ is feasible and therefore $m$ is even. Since $C_{1}$ is a tree, there exists a component $D_{i}$ having a unique edge $f$ in $C_{1}$ joining $D_{i}$ to a vertex in $V\left(C_{1}\right) \backslash V\left(D_{i}\right)$. Then $C_{1} \backslash f$ has exactly two components, each containing an odd number of vertices in $T$ and hence $X \Delta\{e, f\}(=X \backslash\{e, f\})$ is feasible in $(G, T)$.

Now let us consider the case when $e \in Y$. If the subgraph $(V, X \cup\{e\})$ has a cycle containing $e$, then we can pick an edge $f \in X \backslash Y$ in the cycle and $X \Delta\{e, f\}$ is feasible in $(G, T)$. Thus we may assume that $e$ joins two components $C_{1}, C_{2}$ of the subgraph $(V, X)$. If $Y$ has an edge $f$ joining a vertex in $V\left(C_{1}\right) \cup V\left(C_{2}\right)$ to a vertex out of $V\left(C_{1}\right) \cup V\left(C_{2}\right)$, then $X \Delta\{e, f\}(=X \cup\{e, f\})$ is feasible, because the sum of three odd integers is odd. So we may assume that there is a list of components $D_{1}, \ldots, D_{m}$ of the subgraph $(V, Y)$ such that $V\left(D_{1}\right) \cup \cdots \cup V\left(D_{m}\right)=V\left(C_{1}\right) \cup V\left(C_{2}\right)$ and $m$ is even. Since $C_{1} \cup C_{2} \cup e$ is a tree, there exists a component $D_{i}$ having a unique edge $f \in X$ that joins a component $D_{i}$ to a vertex in $V\left(C_{1}\right) \cup V\left(C_{2}\right) \backslash V\left(D_{i}\right)$. We deduce that $X \Delta\{e, f\}(=X \cup\{e\} \backslash\{f\})$ is feasible in $(G, T)$.

Proposition 4.2. If $T=V(G)$, then $\mathcal{G}(G, T)$ is an even binary delta-matroid having $B L(G)$ as a fundamental graph.

If $|T| \leq 1$, then $\mathcal{G}(G, T)$ is the cycle matroid of $G$ (so that each feasible set is a base of the cycle matroid).

Proof. If $T=V(G)$, then, by Proposition 3.2, $X \subseteq E(G)$ is feasible in $\mathcal{G}(G, T)$ if and only if the binary line graph of the subgraph $(V(G), X)$ has the nonsingular adjacency matrix. So $\mathcal{G}(G, T)$ is an even binary delta-matroid.

If $|T| \leq 1$, then every $T$-spanning forest is a spanning forest and therefore each feasible set is a base of the cycle matroid.

\section{GRAFT MINORS}

We now define a graft minor. Let $(G, T)$ be a graft. For an edge $e$ of $G$, we define $(G, T) \backslash e$ to be $(G \backslash e, T)$. This operation is called the deletion of $e$ in a graft $(G, T)$. For an isolated vertex $v$ of $G$, let $(G, T) \backslash v$ be $(G \backslash v, T \backslash\{v\})$. This is the deletion of a vertex $v$. We define $(G, T) / e$ to be $\left(G / e, T^{\prime}\right)$ where $T^{\prime}$ is described as follows. 

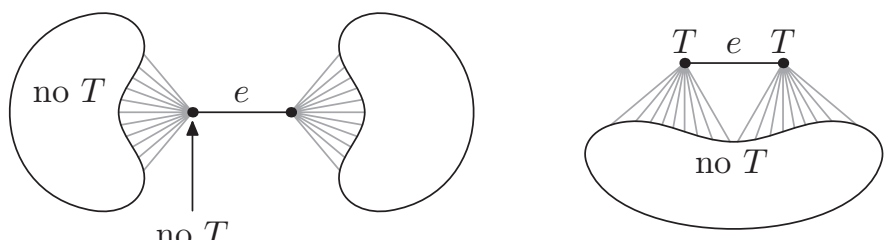

FIGURE 3. T-bridge and T-tunnel.

Let $e^{*}$ be the vertex in $G / e$ obtained by identifying the ends $u$ and $v$ of $e$. Then

$$
T^{\prime}= \begin{cases}(T \backslash\{u, v\}) \cup\left\{e^{*}\right\} & \text { if exactly one of } u \text { or } v \text { is in } T, \\ T \backslash\{u, v\} & \text { otherwise. }\end{cases}
$$

This operation is called the contraction of $e$ in a graft $(G, T)$. Note that if $e$ is a loop, then we define $(G, T) / e=(G, T) \backslash e$. We say that $\left(G^{\prime}, T^{\prime}\right)$ is a minor of a graft $(G$, $T)$ if $\left(G^{\prime}, T^{\prime}\right)$ is obtained from $(G, T)$ by a sequence of deletions and contractions.

Let $\kappa(G, T)$ be the number of components of $G$ having no vertices in $T$. An edge $e$ is a $T$-bridge of a graft $(G, T)$ if $\kappa(G \backslash e, T)>\kappa(G, T)$ (see Fig. 3). We now relate graft minors to delta-matroid minors. Our goal is to show that the set of graphic delta-matroids is minor-closed.

Proposition 5.1. An edge e belongs to every feasible set of $(G, T)$ if and only if $e$ is a T-bridge.

Proof. We may assume that $G$ is connected. If $e$ is a $T$-bridge, then it is clear that every feasible set $F$ of $(G, T)$ contains $e$, because otherwise the graph $(V(G), F)$ has a component $C$ such that $V(C) \cap T=\emptyset$ and $V(C)$ does not span $G$.

Conversely, suppose that $e$ is not a $T$-bridge. So either $e$ is not a cut-edge, or each component of $G \backslash e$ has at least one vertex in $T$. Let $F$ be a feasible set of $(G \backslash e, T)$; such a feasible set always exists, because $\mathcal{G}(G \backslash e, T)$ is a delta-matroid by Proposition 4.1. We wish that $F$ is feasible in $(G, T)$. We may assume that the graph $(V(G), F)$ has a component $C$ such that $V(C) \cap T=\emptyset$ and $V(C)$ spans a component of $G \backslash e$. This implies that $e$ is not a cut-edge. Then $G \backslash e$ is connected and so $V(C)$ spans $G$ and therefore $F$ is feasible in $(G, T)$.

Proposition 5.2. Let $(G, T)$ be a graft. For an edge e of $G$,

$$
\mathcal{G}((G, T) \backslash e)= \begin{cases}\mathcal{G}(G, T) \backslash\{e\} & \text { if e is not a T-bridge } \\ \mathcal{G}(G, T) \Delta\{e\} \backslash\{e\} & \text { otherwise. }\end{cases}
$$

Proof. We may assume that $G$ is connected. Suppose that $e$ is not a $T$ bridge. If $F$ is a feasible set in $(G, T)$ not containing $e$, then it is clear that $F$ is feasible in $(G \backslash e, T)$. Conversely, if $F$ is a feasible set in $(G \backslash e, T)$, then by the Journal of Graph Theory DOI 10.1002/jgt 
proof of Proposition 5.1, $F$ is feasible in $(G, T)$. This proves that $\mathcal{G}((G, T) \backslash e)=$ $\mathcal{G}(G, T) \backslash\{e\}$.

Now it remains to consider the case when $e$ is a $T$-bridge. Let $C_{1}, C_{2}$ be the components of $G \backslash e$ such that $V\left(C_{1}\right) \cap T=\emptyset$. Let $F$ be a feasible set in $(G \backslash e, T)$. Then $F=F_{1} \cup F_{2}$ where $F_{1}, F_{2}$ are feasible sets in grafts $\left(C_{1}, V\left(C_{1}\right) \cap T\right)$, $\left(C_{2}, V\left(C_{2}\right) \cap T\right)$, respectively. Since $V\left(C_{1}\right) \cap T=\emptyset, F \cup\{e\}$ is feasible in $(G$, $T)$. Conversely, every feasible set $F^{\prime}$ in $(G, T)$ can be written as $F^{\prime}=F_{1} \cup F_{2} \cup$ $\{e\}$ where $F_{1}, F_{2}$ are feasible sets in grafts $\left(C_{1}, V\left(C_{1}\right) \cap T\right),\left(C_{2}, V\left(C_{2}\right) \cap T\right)$, respectively. This is because $C_{1}$ has no vertices in $T$ and therefore the component containing $e$ in each feasible set has to span all vertices in $C_{1}$. Thus $\mathcal{G}((G, T) \backslash e)=$ $\mathcal{G}(G, T) \Delta\{e\} \backslash\{e\}$ if $e$ is a $T$-bridge.

An edge $e=u v$ of a graft $(G, T)$ is called a T-tunnel if $V(C) \cap T=\{u, v\}$ for the component $C$ of $G$ containing $e$ (see Fig. 3).

Proposition 5.3. An edge e of $G$ belongs to no feasible set of $(G, T)$ if and only if $e$ is a loop or a T-tunnel.

Proof. We may assume that $G$ is connected. It is obvious that no feasible set in $(G, T)$ contains loops or $T$-tunnels. Now we assume that $e$ is neither a $T$-tunnel nor a loop. We claim that there exists a feasible set containing $e$.

Let $R$ be a spanning tree of $G$ containing $e$. If $|T|$ is odd or $T=\emptyset$, then $E(R)$ is feasible in $(G, T)$. So we may assume that $|T|$ is even and $|T| \geq 2$. Let $R^{\prime}$ be the minimal subtree of $R$ containing all vertices in $T$. Then all leaves of $R^{\prime}$ are in $T$ and $R^{\prime}$ has at least two leaves because $|T| \geq 2$.

If $R^{\prime}$ has at least three leaves, then there is an edge $f$ in $R^{\prime}$ incident with a leaf such that $f \neq e$. Moreover each component of $R \backslash f$ has an odd number of vertices in $T$. So $E(R) \backslash\{f\}$ is feasible in $(G, T)$.

If $R^{\prime}$ has exactly two leaves, then $R^{\prime}$ is a path between vertices in $T$. Since $e$ is not a $T$-tunnel, $\left|E\left(R^{\prime}\right)\right| \geq 2$. Let $f$ be an edge in $R^{\prime}$ other than $e$. Then each component of $R \backslash f$ has exactly one vertex in $T$ and so $E(R) \backslash\{f\}$ is feasible in $(G, T)$.

Proposition 5.4. Let $(G, T)$ be a graft and let e be an edge of $G$. Then

$$
\mathcal{G}((G, T) / e)= \begin{cases}\mathcal{G}(G, T) \Delta\{e\} \backslash\{e\} & \text { if e is neither a } T \text {-tunnel nor a loop } \\ \mathcal{G}(G, T) \backslash\{e\} & \text { otherwise. }\end{cases}
$$

Proof. We may assume that $G$ is connected. We may assume that $e$ is not a loop. If $e$ is a loop, then we use Proposition 5.2.

It is straightforward to show that, if $e$ is a $T$-tunnel, then the set of feasible sets in $(G, T)$ is exactly the set of edge-sets of spanning trees of $G / e$. Thus $\mathcal{G}((G, T) / e)=$ $\mathcal{G}(G / e, \emptyset)=\mathcal{G}(G, T) \backslash\{e\}$.

We may now assume that $e$ is not a $T$-tunnel. Let $T^{*}$ be the set of vertices of $G / e$ such that $(G, T) / e=\left(G / e, T^{*}\right)$. It is trivial to show that if $F^{\prime}$ is a feasible set in $(G$, $T)$ containing $e$, then $F^{\prime} \backslash\{e\}$ is feasible in $(G, T) / e$. We claim that if $F$ is a feasible 
set in $(G, T) / e$, then $F \cup\{e\}$ is feasible in $(G, T)$. This claim is trivial if $T^{*} \neq \varnothing$. If $T^{*}=\emptyset$, then $F$ is an edge-set of a spanning tree of $G / e$. Since $e$ is not a $T$-tunnel, $T=\varnothing$ and therefore $F \cup\{e\}$ is an edge-set of a spanning tree of $G$ and $F \cup\{e\}$ is feasible in $(G, T)$. Therefore, $\mathcal{G}((G, T) / e)=\mathcal{G}(G, T) \Delta\{e\} \backslash\{e\}$.

Proposition 5.5. Let $(G, T)$ be a graft and let $v$ be an isolated vertex of $G$. Then

$$
\mathcal{G}((G, T) \backslash v)=\mathcal{G}(G \backslash v, T \backslash\{v\})
$$

Proof. This proof is easy and so omitted.

Theorem 5.6. If a graft $\left(G^{\prime}, T^{\prime}\right)$ is a minor of a graft $(G, T)$, then a graphic delta-matroid $\mathcal{M}^{\prime}=\mathcal{G}\left(G^{\prime}, T^{\prime}\right) \Delta X^{\prime}$ is a minor of a graphic delta-matroid $\mathcal{M}=$ $\mathcal{G}(G, T) \Delta X$.

Proof. The theorem follows from Propositions 5.2, 5.4, and 5.5.

Theorem 5.7. A minor of a graphic delta-matroid is graphic.

Proof. Let us show that if $\mathcal{M}_{1}=\left(E_{1}, \mathcal{F}_{1}\right)$ is a minor of a graphic delta-matroid $\mathcal{M}_{2}=\left(E_{2}, \mathcal{F}_{2}\right)$, then $\mathcal{M}_{1}$ is graphic. We will proceed by induction on $\left|E_{1} \backslash E_{2}\right|$. If $E_{1}=E_{2}$, then $\mathcal{M}_{1}=\mathcal{M}_{2} \Delta X$ for some $X \subseteq E_{2}$. So $\mathcal{M}_{1}$ is graphic. If $E_{1} \neq E_{2}$, we may assume that $\left|E_{2} \backslash E_{1}\right|=1$. Let $E_{2} \backslash E_{1}=\{e\}$. By twisting, we may assume that $\mathcal{M}_{2}=\mathcal{G}(G, T)$ for a graft $(G, T)$. Without loss of generality, we may assume that either $\mathcal{M}_{1}=\mathcal{M}_{2} \Delta\{e\} \backslash\{e\}$ or $\mathcal{M}_{1}=\mathcal{M}_{2} \backslash\{e\}$. Since $\mathcal{M}_{1}$ is a delta-matroid, $\mathcal{F}_{1}$ is nonempty.

If $e$ is a $T$-tunnel or a loop, then $\mathcal{M}_{1}=\mathcal{M}_{2} \backslash\{e\}$ because no feasible set of $\mathcal{M}_{2}$ contains $e$ by Proposition 5.3 and $\mathcal{F}_{1}$ is nonempty. So $\mathcal{M}_{1}=\mathcal{G}((G, T) / e)$.

If $e$ is a $T$-bridge, then $\mathcal{M}_{1}=\mathcal{M}_{2} \Delta\{e\} \backslash\{e\}$ because every feasible set of $\mathcal{M}$ contains $e$ by Proposition 5.1. So $\mathcal{M}_{1}=\mathcal{G}((G, T) \backslash e)$.

Otherwise, $\mathcal{M}_{1}$ is either $\mathcal{G}((G, T) / e)$ or $\mathcal{G}((G, T) \backslash e)$.

Corollary 5.8. A graphic delta-matroid is even binary.

Proof. It is enough to prove that, for a graft $(G, T), \mathcal{G}(G, T)$ is an even binary delta-matroid. Suppose that $X=V(G) \backslash T=\left\{v_{1}, v_{2}, \ldots, v_{k}\right\}$. ( $k$ may be 0 .) Then let $X^{\prime}=\left\{v_{1}^{\prime}, v_{2}^{\prime}, \ldots, v_{k}^{\prime}\right\}$ be a set of $k$ elements such that $X^{\prime} \cap V(G)=$ $\emptyset$. Now we define the graph $G^{\prime}$ on $V(G) \cup X^{\prime}$ from $G$ by adding $k$ new vertices in $X^{\prime}$ into $G$ and adding $k$ new edges $e_{1}=v_{1} v_{1}^{\prime}, e_{2}=v_{2} v_{2}^{\prime}, \ldots, e_{k}=$ $v_{k} v_{k}^{\prime}$. Then $(G, T)=\left(G^{\prime}, V\left(G^{\prime}\right)\right) / e_{1} / e_{2} / \cdots / e_{k}$. By Theorem 5.6, $\mathcal{G}(G, T)$ is a minor of $\mathcal{G}\left(G^{\prime}, V\left(G^{\prime}\right)\right)$. By Proposition $4.2, \mathcal{G}\left(G^{\prime}, V\left(G^{\prime}\right)\right)$ is even binary. Since all minors of an even binary delta-matroid are even binary, $\mathcal{G}(G, T)$ is even binary. 


\section{BRANCH-WIDTH AND RANK-WIDTH}

Let $(G, T)$ be a graft and let $\Gamma$ be a fundamental graph of the graphic delta-matroid $\mathcal{G}(G, T)$. In this section, we would like to find a connection between the rank-width of $\Gamma$ and the branch-width of $G$. We note that the choice of $\Gamma$ is independent of the rank-width of $\Gamma$. The reason is that, if $\Gamma$ and $\Gamma^{\prime}$ are fundamental graphs of an even binary delta-matroid, then $\Gamma^{\prime}$ is obtainable from $\Gamma$ by repeatedly applying pivoting and pivoting preserves the cut-rank function, see Oum [15]. Hence we define, for subsets $X$ of $E(G), \rho_{(G, T)}(X)$ to be the cut-rank function $\rho_{\Gamma}(X)$ of a fundamental graph $\Gamma$ of $\mathcal{G}(G, T)$.

Lemma 6.1. Let $(G, T)$ be a graft. If $X$ is a subset of $E(G)$, then $\rho_{(G, T)}(X) \leq \beta_{G}(X)$.

Proof. Suppose the lemma is false. Pick a counterexample $(G, T)$ and $X$ so that $\beta_{G}(X)$ is minimum and among those, $|T|+3|V(G) \backslash T|$ is minimum. Clearly $X \neq \emptyset$ and $X \neq V(G)$.

First we claim that $T=V(G)$. Suppose $v \in V(G) \backslash T$. We may assume that $v$ is incident with an edge in $E(G) \backslash X$ or $v$ is an isolated vertex in $G$; otherwise we replace $X$ with $E(G) \backslash X$. Let $G^{\prime}$ be the graph obtained by adding a new vertex $v^{\prime}$ and a new edge $v v^{\prime}$ to $G$ (Fig. 4) and let $T^{\prime}=T \cup\left\{v, v^{\prime}\right\}$. Then $\beta_{G^{\prime}}(X)=$ $\beta_{G}(X)$ and $\left|T^{\prime}\right|+3\left|V\left(G^{\prime}\right) \backslash T^{\prime}\right|=|T|+3|V(G) \backslash T|-1$. Since $\left(G^{\prime}, T^{\prime}\right)$ is not a counterexample, $\rho_{\left(G^{\prime}, T^{\prime}\right)}(X) \leq \beta_{G^{\prime}}(X)$. Since $(G, T)$ is a minor of $\left(G^{\prime}, T^{\prime}\right)$, $\rho_{(G, T)}(X) \leq \rho_{\left(G^{\prime}, T^{\prime}\right)}(X)$. So $\rho_{(G, T)}(X) \leq \beta_{G}(X)$; a contradiction. This proves that $T=V(G)$.

Let $M$ be the set of vertices meeting both $X$ and $E(G) \backslash X$. By definition, $\beta_{G}(X)=|M|$. We claim that no edges have both ends in $M$. Suppose that there is an edge $e$ of $G$ joining two vertices in $M$. We may assume that $e \notin X$ by replacing $X$ with $E(G) \backslash X$ if necessary. Then $\beta_{G / e}(X)=\beta_{G}(X)-1$. By assumption, $\rho_{(G, T) / e}(X) \leq \beta_{G / e}(X)$. Since there exist fundamental graphs $\Gamma$ and $\Gamma^{\prime}$ of $\mathcal{G}(G, T)$ and $\mathcal{G}\left((G, T) / e\right.$ ), respectively, such that $\Gamma^{\prime}=\Gamma \backslash e$ (Note that $e$ is a vertex of $\Gamma)$, we have $\rho_{(G, T)}(X)=\rho_{\Gamma}(X) \leq \rho_{\Gamma^{\prime}}(X)+1=\rho_{(G, T) / e}(X)+1$. Therefore $\rho_{(G, T)}(X) \leq \beta_{G}(X)$; a contradiction. We conclude that $M$ induces no edges in $G$.

Let $M=\left\{a_{1}, a_{2}, \ldots, a_{k}\right\}$. Since $T=V(G), B L(G)$ is a fundamental graph of $\mathcal{G}(G, T)$ by Proposition 4.2. For $i \in\{1,2, \ldots, k\}$, let $X_{i}$ and $Y_{i}$ be the sets of nonloop edges in $X$ and $E(G) \backslash X$, respectively, incident with $a_{i}$. We know that $e \in X_{i}$

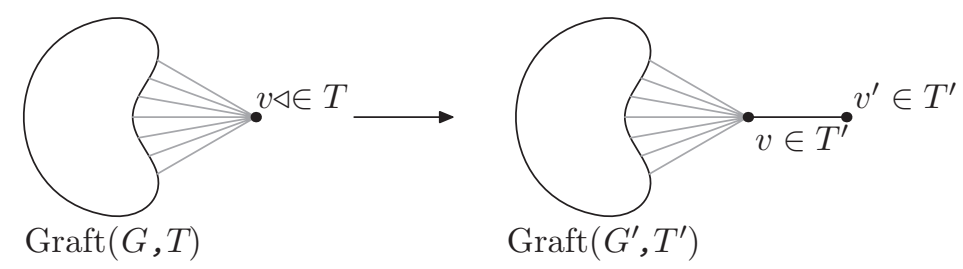

FIGURE 4. Illustration of proof of Lemma 6.1. 
and $f \in Y_{j}$ are adjacent in $B L(G)$ if and only if $i=j$. Therefore

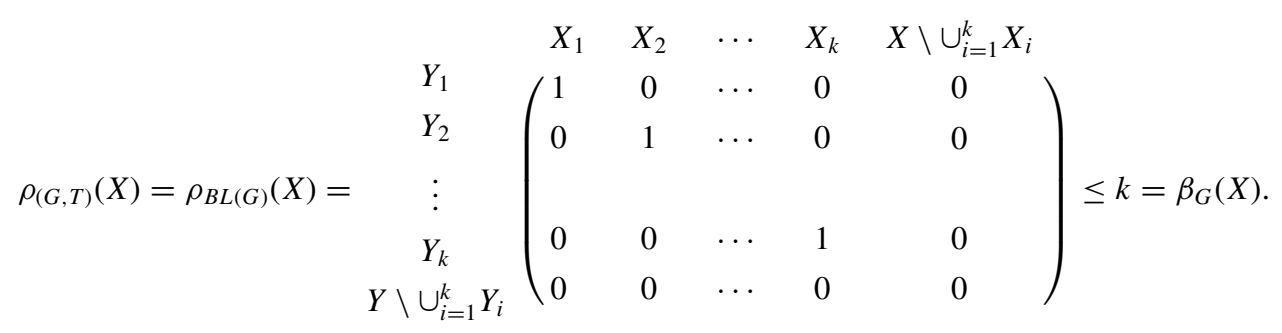

We have an inequality here because some $X_{i}$ or $Y_{i}$ may be empty. 1 and 0 denote block matrices of which every entry is 1 and 0 , respectively.

For a graft $(G, T)$ and a fundamental graph $\Gamma$ of $\mathcal{G}(G, T)$, we will prove that if the rank-width of $\Gamma$ is small, then the branch-width of $G$ is small. Recall that $\lambda_{\mathcal{M}(G)}$ is the matroid connectivity function defined as $\lambda_{\mathcal{M}(G)}(X)=r(X)+r(E(G) \backslash X)-$ $r(E(G))+1$, where $r$ is the rank function of the cycle matroid $\mathcal{M}(G)$ of $G$.

Lemma 6.2. Let $(G, T)$ be a graft. If $G$ is connected, and $|T|$ is odd or $|T|=0$, then $\lambda_{\mathcal{M}(G)}(X)-1 \leq \rho_{(G, T)}(X)$ for all subsets $X$ of $E(G)$.

Proof. Let $F$ be the edge-set of a spanning tree of $G$. Let $Y=E(G) \backslash X$. We assume that $X, Y \neq \emptyset$. We assume that

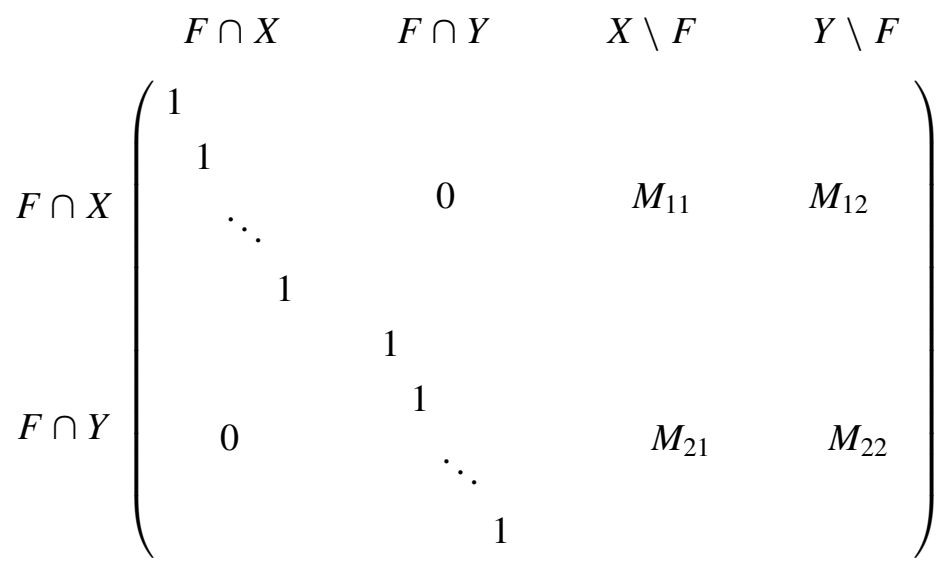

is a binary matrix representation of the cycle matroid $\mathcal{M}(G)$ of $G$. In other words, a set of edges is independent in $\mathcal{M}(G)$ if and only if the corresponding column vectors are linearly independent. The matrix $M=\left(\begin{array}{ll}M_{11} & M_{12} \\ M_{21} & M_{22}\end{array}\right)$ is determined as follows: for $x \in F$ and $y \in E(G) \backslash F$, the $(x, y)$ entry of $M$ is 1 if and only if $x$ is in the fundamental cycle of $y$ with respect to the spanning tree $(V(G), F)$ of $G$. Now, $r(X)=|F \cap X|+\operatorname{rank}\left(M_{21}\right), r(Y)=|F \cap Y|+\operatorname{rank}\left(M_{12}\right), r(E(G))=|F|$, and therefore $\lambda_{\mathcal{M}(G)}(X)=\operatorname{rank}\left(M_{21}\right)+\operatorname{rank}\left(M_{12}\right)+1$. 
Since $|T|$ is odd or $|T|=0, F$ is feasible in $(G, T)$. Let $\Gamma$ be the fundamental graph of $\mathcal{G}(G, T)$ with respect to $F$. Then $\rho_{(G, T)}(X)=\operatorname{rank}(N)$ where

$$
N=A(\Gamma)[X, Y]=\begin{aligned}
& F \cap Y \\
& F \cap X \backslash F \\
& X \backslash F
\end{aligned}\left(\begin{array}{ll}
N_{11} & N_{12} \\
N_{21} & N_{22}
\end{array}\right)
$$

Since $F$ is maximally feasible in $\mathcal{G}(G, T), F \Delta\{x, y\}$ is not feasible when $x, y \notin$ $F$ and therefore $N_{22}=0$. By definition, $N_{12}=M_{12}$ and $N_{21}=\left(M_{21}\right)^{t}$. We have the following inequality: $\operatorname{rank}(N) \geq \operatorname{rank}\left(N_{21}\right)+\operatorname{rank}\left(N_{12}\right)=\operatorname{rank}\left(M_{21}\right)+$ $\operatorname{rank}\left(M_{12}\right)=\lambda_{\mathcal{M}(G)}-1$.

Lemma 6.3. Let $(G, T)$ be a graft. If $G$ is connected, then $\lambda_{\mathcal{M}(G)}(X)-2 \leq$ $\rho_{(G, T)}(X)$ for all subsets $X$ of $E(G)$.

Proof. If $|T|$ is odd or $T=\emptyset$, then the lemma is implied by Lemma 6.2. So we may assume that $|T|$ is even and $|T| \geq 2$.

Let $v$ be a vertex of $G$. Let $G^{\prime}$ be the graph obtained from $G$ by adding a new vertex $v^{\prime}$ and a new edge $v v^{\prime}$. Let $T^{\prime}=T \cup\left\{v^{\prime}\right\}$. Then $\left(G^{\prime}, T^{\prime}\right)$ is a graft such that $\left|T^{\prime}\right|$ is odd. Moreover $(G, T)$ is a minor of $\left(G^{\prime}, T^{\prime}\right)$, obtained by deleting $v v^{\prime}$ and deleting $v^{\prime}$. Then $\lambda_{\mathcal{M}\left(G^{\prime}\right)}(X)=\lambda_{\mathcal{M}(G)}(X)$. By Lemma 6.2, $\lambda_{\mathcal{M}\left(G^{\prime}\right)}(X)-$ $1 \leq \rho_{\left(G^{\prime}, T^{\prime}\right)}(X)$. Let $\Gamma$ be a fundamental graph of $\mathcal{G}(G, T)$. Then there exists a fundamental graph $\Gamma^{\prime}$ of $\mathcal{G}\left(G^{\prime}, T^{\prime}\right)$ such that $\Gamma=\Gamma^{\prime} \backslash v^{\prime}$. So $\rho_{\left(G^{\prime}, T^{\prime}\right)}(X)=\rho_{\Gamma^{\prime}}(X) \leq$ $\rho_{\Gamma}(X)+1=\rho_{(G, T)}(X)+1$. Therefore $\lambda_{\mathcal{M}(G)}(X)-2 \leq \rho_{(G, T)}(X)$.

Hicks and McMurray [10] and Mazoit and Thomassé [14] independently proved the following theorem.

Theorem 6.4 (Hicks and McMurray [10]). Let $G$ be a graph with at least one cycle. Then the branch-width of $G$ is equal to the branch-width of $\mathcal{M}(G)$.

Theorem 6.5. Let $(G, T)$ be a graft. Let $\Gamma$ be a fundamental graph of $\mathcal{G}(G, T)$. If the branch-width of $G$ is $k$, then the rank-width of $\Gamma$ is $k, k-1$, or $k-2$.

Proof. We may assume that $G$ is connected. By Lemma 6.1, the rank-width of $\Gamma$ is at most $k$. If $G$ has a cycle, then the cycle matroid of $G$ has the branch-width $k$ by Theorem 6.4. Then by Lemma 6.3, the rank-width of $\Gamma$ is at least $k-2$. If $G$ is a tree, then the branch-width of $G$ is at most 2 . Then the theorem is implied because the rank-width of $\Gamma$ is nonnegative.

The previous theorem implies that for a graph $G$, the rank-width of $B L(G)$ is $k, k-1$, or $k-2$ when $k$ is the branch-width of $G$. Consequently, if $G$ is a simple graph, then the rank-width of the line graph $L(G)$ is at most the branch-width of $G$. What could happen if $G$ is not a simple graph? 
Corollary 6.6. Let $G$ be a graph. If the branch-width of $G$ is $k$, then the rank-width of $L(G)$ is $k, k-1$, or $k-2$.

Proof. Suppose not. Let $G$ be a counterexample with minimum number of loops and parallel edges. If $G$ is a simple graph, then it is implied by Theorem 6.5 when $T=V(G)$.

If $G$ has a loop $e$, then we consider another graph $G^{\prime}$ obtained by adding a new vertex $v^{\prime}$ and replacing $e$ by a new edge joining the end of $e$ with $v^{\prime}$. Then $L(G)=L\left(G^{\prime}\right)$. Since the number of loops in $G^{\prime}$ is less than the number of loops in $G$, the rank-width of $L\left(G^{\prime}\right)$ is $k, k-1$, or $k-2$; a contradiction because $G$ and $G^{\prime}$ have the same branch-width. So $G$ has no loops.

Thus, $G$ has an edge $e$ parallel to $f$. Unless the branch-width of $G \backslash e$ is $1, G \backslash e$ and $G$ have the same branch-width. If the branch-width of $G \backslash e$ is 1 , then the branchwidth of $G$ is 2 . In both cases, the rank-width of $L(G \backslash e)$ is $k, k-1$, or $k-2$. In order to obtain $L(G)$ from $L(G \backslash e)$, we can add a vertex $e$ to $L(G \backslash e)$ so that $e$ and $f$ have the same set of neighbors and $e$ and $f$ are adjacent. This operation (creating twins) preserves the rank-width, as was shown by Oum [15]. So the rank-width of $L(G)$ is equal to the rank-width of $L(G \backslash e)$; a contradiction.

\section{OBTAINING THE GRID MINOR}

We are now going towards the final theorem. The $n \times n$ grid is a graph on the vertex set $\left\{(i, j) \in \mathbb{Z}^{2}: 0 \leq i<n, 0 \leq j<n\right\}$ such that two vertices $(a, b)$ and $(c$, $d$ ) are adjacent if and only if $|a-c|+|b-d|=1$. The following lemma is a direct consequence of Robertson and Seymour's theorem on graphs of large tree-width.

Lemma 7.1. For each positive integer $n$, there is a constant $c$ such that if the rank-width of a fundamental graph of the graphic delta-matroid of a graft $(G, T)$ is larger than $c$, then $(G, T)$ has a minor a minor $\left(G^{\prime}, T^{\prime}\right)$ such that $G^{\prime}$ is isomorphic to the $3 n \times 3 n$ grid.

Proof. Let $N$ be a constant so that if the branch-width of a graph $G$ is larger than $N$ then $G$ contains a minor isomorphic to the $3 n \times 3 n$ grid. By Theorem 6.5, if the rank-width of a fundamental graph of $\mathcal{G}(G, T)$ is larger than $N$, then the branchwidth of $G$ is larger than $N$ and therefore $G$ has a minor isomorphic to the $3 n \times 3 n$ grid. Since every graph minor operation corresponds to a graft minor operation by Propositions 5.2, 5.4, and 5.5, we deduce that $(G, T)$ has a minor $\left(G^{\prime}, T^{\prime}\right)$ such that $G^{\prime}$ is isomorphic to the $3 n \times 3 n$ grid.

In Lemma 7.1, we obtained a graft $(G, T)$ such that $G$ is the $3 n \times 3 n$ grid, but $T$ could be arbitrary. We aim to obtain a minor $\left(G^{\prime}, T^{\prime}\right)$ of $(G, T)$ such that $G^{\prime}$ is an $n \times n$ grid and $T^{\prime}=\emptyset$.

Lemma 7.2. Let $G=(V, E)$ be the $3 n \times 3 n$ grid. Let $T$ be a subset of $V$. Then $(G$, $T)$ has a minor $\left(G^{\prime}, T^{\prime}\right)$ such that $G^{\prime}$ is isomorphic to the $n \times n$ grid and $T^{\prime}=\emptyset$. 
Proof. We will partition $V$ into $3 \times 3$ blocks, delete at most one vertex from each block, and then contract each block to a vertex. Each block consists of vertices $(3 i+a, 3 j+b)$ where $a, b \in\{0,1,2\}$.

If a block has an even number of vertices in $T$, then we do not delete any vertex in the block. We contract the block into a vertex $v$ and then $v$ will not belong to $T$.

If a block has an odd number of vertices in $T$, then we pick one vertex $w$ in $T$ and delete all edges incident with $w$ and then delete $w$. After removal of $w$, the block is still connected because the $3 \times 3$ grid is 2 -connected. Furthermore it has an even number of vertices in $T$. We now contract the block into a single vertex and delete all loops and parallel edges.

It is evident that after treating each block, we get $n \times n$ vertices. It remains to show that edges are present as in the $n \times n$ grid. In the $3 n \times 3 n$ grid, there are three edges connecting two adjacent blocks. We remove at most one vertex from each block and therefore at least one edge remains between pairs of adjacent blocks. So the graph is indeed isomorphic to the $n \times n$ grid.

\section{EXCLUDING A BIPARTITE CIRCLE GRAPH FROM LINE GRAPHS}

Now we are ready to prove our main theorem.

Theorem 8.1. Let $H$ be a bipartite circle graph. Then there is a constant $c(H)$ such that if a fundamental graph $\Gamma$ of $\mathcal{G}(G, T)$ for a graft $(G, T)$ has the rank-width larger than $c(H)$, then $\Gamma$ has a pivot-minor isomorphic to $H$.

We will need the following two lemmas.

Lemma 8.2 (de Fraysseix [5]). A simple graph is a bipartite circle graph if and only if it is a fundamental graph of a planar matroid.

Lemma 8.3 (Robertson et al. [19, (1.5)]). If $H$ is a planar graph such that $|V(H)|+2|E(H)| \leq n$, then $H$ is isomorphic to a minor of the $2 n \times 2 n$ grid.

Proof of Theorem 8.1. By Lemma 8.2, $H$ is a fundamental graph of the cycle matroid of a planar graph $J$ and so $H$ is a fundamental graph of $\mathcal{G}(J, \varnothing)$. Let $n$ be the minimum number so that $J$ is isomorphic to a minor of the $n \times n$ grid $G_{n}$. (By Lemma 8.3, the number $n$ exists.) It follows that $(J, \varnothing)$ is isomorphic to a minor of $\left(G_{n}, \varnothing\right)$.

By Lemma 7.1, there is a constant $c$ such that, if the rank-width of a fundamental graph $\Gamma$ of $\mathcal{G}(G, T)$ for a graft $(G, T)$ is larger than $c$, then $(G, T)$ has a minor $\left(G^{\prime}\right.$, $\left.T^{\prime}\right)$ such that $G^{\prime}$ is isomorphic to the $3 n \times 3 n$ grid. By Lemma 7.2, $\left(G^{\prime}, T^{\prime}\right)$ has a minor isomorphic to $\left(G_{n}, \emptyset\right)$.

Since $\left(G_{n}, \emptyset\right)$ has a minor isomorphic to $(J, \emptyset),(G, T)$ has a minor isomorphic to $(J, \varnothing)$. It follows that $\Gamma$ has a pivot-minor isomorphic to $H$.

The above theorem implies that, for a fixed bipartite circle graph $H$, every binary line graph of sufficiently large rank-width must have a pivot-minor isomorphic to $H$. In order to prove this for line graphs, it remains to consider non-simple graphs. 
Theorem 8.4. Let $H$ be a bipartite circle graph. There is a constant $c(H)$ such that if the line graph $L(G)$ has the rank-width larger than $c(H)$, then $L(G)$ has a pivot-minor isomorphic to $H$.

Proof. It is enough to consider a non-simple graph $G$ by Theorem 8.1 and the proof is almost identical to the proof of Corollary 6.6. We omit the detail.

\section{EXCLUDING A BIPARTITE CIRCLE GRAPH FROM CIRCLE GRAPHS}

Conjecture 1.1 is verified for line graphs in Theorem 8.4 and for bipartite graphs by Oum [15]. In this section, we would like to present how the following theorem of Johnson [11] implies Conjecture 1.1 for circle graphs.

Theorem 9.1 (Johnson [11, Theorem 2.5]). Let $n$ be a positive integer. There is a positive integer $t$ such that if $D$ is an Eulerian digraph with tree-width at least $t$, then the medial grid of size $n$ is immersed in $D$.

We need a few definitions to decode this theorem properly. An Eulerian digraph is a digraph such that the in-degree is equal to the out-degree at each vertex. For our purpose it is enough to consider a 4-regular Eulerian digraph such that it is connected and each vertex has in-degree 2 and out-degree 2, even though Johnson's theorem works for general Eulerian digraphs.

Unlike the usual tree-width of graphs, the tree-width of an Eulerian digraph [11, page 8] is defined more like branch-width. In fact, the tree-width of an Eulerian digraph $D$ is defined to be the branch-width of the following symmetric submodular function $\kappa: 2^{V(D)} \rightarrow \mathbb{Z}$, where $\kappa(X)=|\delta(X)|$, that is the number of non-loop edges having exactly one end in $X$.

The medial grid of size $n$ is the medial graph of the $n \times n$ grid with all edges directed clockwise around the vertices of the grid (see Fig. 5).

An Eulerian digraph $H$ is immersed in an Eulerian digraph $D$ if $H$ is obtained from $D$ by repeatedly applying the following operations [11, Theorem 2.2]:

(i) Deleting a vertex of out-degree 0 ,

(ii) Deleting a loop,

(iii) (Suppressing) Contracting an edge incident to a vertex of out-degree 1,

(iv) (Splitting) Replacing a vertex $v$ by two new vertices $v_{1}$ and $v_{2}$ and partitioning the edges incident with $v$ between $v_{1}$ and $v_{2}$ so that each of $v_{1}$ and $v_{2}$ has the same in-degree and out-degree (see Fig. 6).

We will sketch how to deduce Conjecture 1.1 for circle graphs from Theorem 9.1. We need understand the following four ingredients:

(a) how 4-regular Eulerian digraphs are associated to circle graphs,

(b) why medial grids are associated to fundamental graphs of the cycle matroid of the grid, 


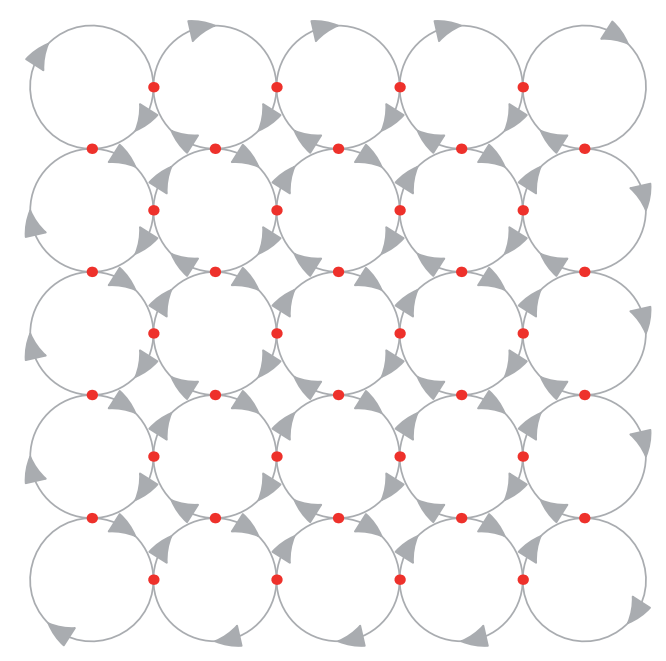

FIGURE 5. Medial grid of size 5 .
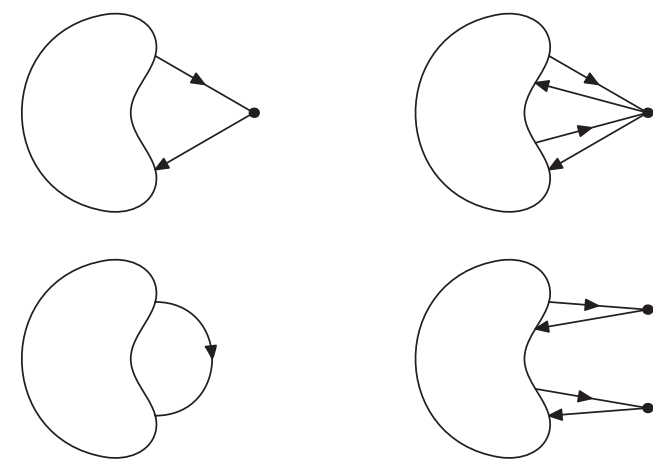

FIGURE 6. Suppressing (left) and splitting (right).

(c) why immersions are related to pivot-minors of associated circle graphs, and

(d) how the tree-width of a 4-regular Eulerian digraph is related to the rank-width of associated circle graphs.

For (a) and (b), see Figures 7 and 8, respectively. Bouchet [1] showed that the Eulerian circuits form a delta-matroid, which he calls an Eulerian delta-matroid. It is straightforward to check the a minor of an Eulerian delta-matroid corresponds to an immersion of a 4-regular Eulerian digraph. This will verify (c).

To see (d), we will show that if a 4-regular Eulerian digraph has small tree-width, then its associated circle graph has small rank-width. We warn that the notion of tree-width for 4-regular Eulerian digraphs in [11] is different from the usual treewidth or the directed tree-width [12]. 

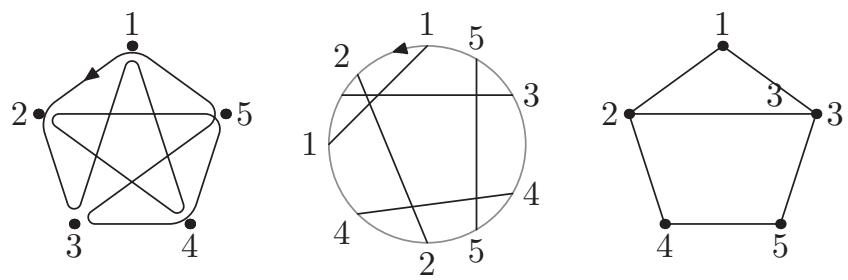

FIGURE 7. 4-Regular Eulerian digraphs, chord diagrams, and circle graphs.
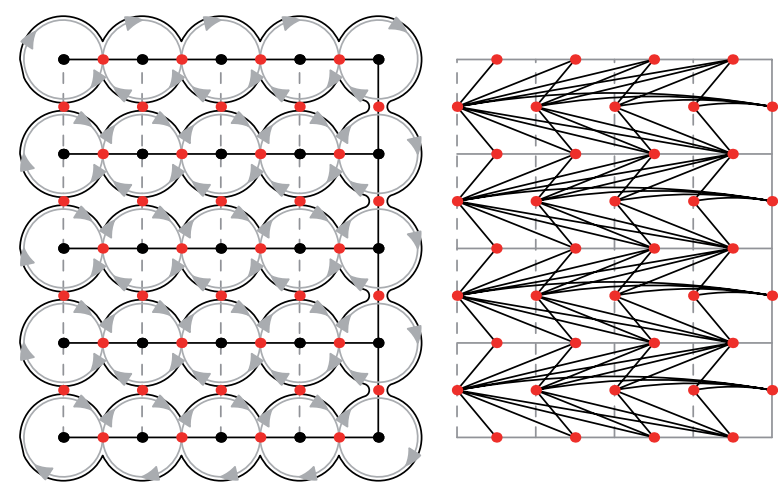

FIGURE 8. A medial grid and a fundamental graph of the cycle matroid of the grid.

Lemma 9.2. Let $D$ be a 4-regular Eulerian digraph and let $G$ be the associated circle graph with respect to an Eulerian circuit $C$ of $D$. If the tree-width of $D$ is $2 t$, then the rank-width of $G$ is at most $t(t-1) / 2$.

Proof. Let $(T, \mu)$ be a tree-decomposition of $D$ of width $2 t$, which is a branchdecomposition of $\kappa_{D}$ or a rank-decomposition of $G$. (So $T$ is a subcubic tree and $\mu$ is a bijection from $V(G)=V(D)$ to the set of leaves of $D$.) We claim that the width of the rank-decomposition $(T, \mu)$ is at most $t(t-1) / 2$.

To prove the claim, it is enough to show that, for a subset $X$ of $V(D)$, if $\kappa(X)=2 k$ then $\rho_{G}(X) \leq k(k-1) / 2$. Because $D$ has an Eulerian circuit, $\left|\delta^{+}(X)\right|=\left|\delta^{-}(X)\right|$ and so $\kappa(X)=|\delta(X)|$ is always even. Let $M$ be the chord diagram of $G$ corresponding to $D$ so that the circle corresponds to the Eulerian circuit $C$. By deleting $2 k$ edges in $\delta(X)$, we obtain $2 k$ segments of the circle in $M$. (A segment can be a single point.)

We color $k$ segments having vertices in $X$ by blue and other $k$ segments by red. Then the colors of segments of the circle of $M$ alternate between red and blue. We color each chord of $M$ by blue if it belongs to $X$ and red otherwise. In the chord diagram $M$, the color of each chord is equal to the color of segments that it meets. The cut-rank function $\rho_{G}(X)$ depends on the adjacency of blue and red chords. For distinct $i, j \in\{1,2, \ldots, k\}$, let $R_{i, j}$ be the set of red chords of $M$ incident with the $i$ th and the $j$ th segments. It is easy to observe that for each blue chord $x$, the set of 
red chords intersecting with $x$ is the disjoint union $\cup_{(i, j) \in L} R_{i, j}$ for some $L$. Therefore $\rho_{G}(X) \leq\left(\begin{array}{c}k \\ 2\end{array}\right)$.

Combining all the above arguments, we obtain the following theorem.

Theorem 9.3. For a bipartite circle graph $H$, there is a constant $c(H)$ such that if a circle graph $G$ has rank-width larger than $c(H)$, then $G$ has a pivot-minor isomorphic to $H$.

Proof. Suppose $G$ is a circle graph with sufficiently large rank-width. Let $D$ be the corresponding 4-regular Eulerian digraph. Then, by Lemma 9.2, $D$ has large treewidth. Theorem 9.1 implies that $D$ immerses a large medial grid. So a fundamental graph of a grid is a pivot-minor of $G$. By Lemmas 8.2 and 8.3, a fundamental graph of a large grid has a pivot-minor isomorphic to $H$.

\section{ACKNOWLEDGMENTS}

I am very grateful to Jim Geelen for insightful discussions. His contributions were substantial. I also thank referees for their careful and helpful suggestions, which help me improve this article significantly. This work was partially supported by NSF grant 0354742 and the SRC Program of Korea Science and Engineering Foundation (KOSEF) grant funded by the Korea Government (MOST) (No. R11-2007-03501002-0).

\section{REFERENCES}

[1] A. Bouchet, Greedy algorithm and symmetric matroids, Math Programming 38(2) (1987), 147-159.

[2] A. Bouchet, Representability of $\Delta$-matroids, In Combinatorics (Eger, 1987), volume 52 of Colloq. Math. Soc. János Bolyai. North-Holland, Amsterdam, 1988 ,

[3] A. Bouchet, Circle graph obstructions, J Combin Theory Ser B 60(1) (1994), 107-144.

[4] B. Courcelle and S. Olariu, Upper bounds to the clique width of graphs, Discrete Appl Math 101(1-3) (2000), 77-114.

[5] H. de Fraysseix, Local complementation and interlacement graphs, Discrete Math 33(1) (1981), 29-35.

[6] N. Deo, M. S. Krishnamoorthy, and A. B. Pai, Generalizations of line graphs and applications, Information Processing Lett 6(1) (1977), 14-17.

[7] M. Doob, An interrelation between line graphs, eigenvalues, and matroids, J Combin Theory Ser B 15 (1973), 40-50.

[8] J. Geelen, B. Gerards, and G. Whittle, Excluding a planar graph from GF( $q$ )representable matroids, J Combin Theory Ser B 97(6) (2007), 971-998. 
[9] F. Gurski and E. Wanke, Line graphs of bounded clique-width, Discrete Math 307(22) (2007), 2734-2754.

[10] I. V. Hicks and N. B. McMurray, Jr., The branchwidth of graphs and their cycle matroids, J Combin Theory Ser B 97(5) (2007), 681-692.

[11] C. D. T. Johnson, Eulerian digraph immersion, $\mathrm{PhD}$ thesis, Princeton University, 2002.

[12] T. Johnson, N. Robertson, P. Seymour, and R. Thomas, Directed tree-width, J Combin Theory Ser B 82(1) (2001), 138-154.

[13] G. Kishi and Y. Uetake, Rank of edge incidence matrix, IEEE Trans Circuit Theory CT-16 (1969), 230-232.

[14] F. Mazoit and S. Thomassé, Branchwidth of graphic matroids, In A. Hilton and J. Talbot (Editors), Surveys in combinatorics 2007, Vol. 346 of London Math. Soc. Lecture Note Ser., Cambridge University Press, Cambridge, UK, 2007, pp. 275-286.

[15] S. Oum, Rank-width and vertex-minors, J Combin Theory Ser B 95(1) (2005), $79-100$.

[16] S. Oum and P. Seymour, Approximating clique-width and branch-width, J Combin Theory Ser B 96(4) (2006), 514-528.

[17] J. G. Oxley, Matroid theory, Oxford University Press, New York, 1992.

[18] N. Robertson and P. Seymour, Graph minors. X. Obstructions to treedecomposition, J Combin Theory Ser B 52(2) (1991), 153-190.

[19] N. Robertson, P. Seymour, and R. Thomas, Quickly excluding a planar graph, J Combin Theory Ser B 62(2) (1994), 323-348.

[20] A. W. Tucker, A combinatorial equivalence of matrices. In R. Bellman and M. Hall, Jr. (Editors), Combinatorial analysis, American Mathematical Society, Providence, RI, pp. 129-140. 1960. 